\title{
Introduction: hybrid networks and political domains
}

To most people the global politics of the Internet is simply off the radar. We tend to think of the Internet as yet another medium able to transmit a wide variety of messages, data and cultural products very quickly, a technological innovation allowing stock traders, architects and activists to work in new ways, or as a new social space, where we can connect with old and new friends. But the Internet is not just a medium, a technology and a social space (Cavanagh 2007: 4). It is also a new political domain. For instance, the news regularly reports on the use of filtering in China, Internet users may think about getting caught if they download music and films illegally, and parents wonder if enough is done to keep their kids safe online. But we rarely think of such scattered issues as political, and as long as the Internet works, we have more pressing regulatory, legal and safety concerns to worry about. Nevertheless, what started as a technical experiment connecting a small number of computers in US universities (Abbate 1999) has now become a crucial infrastructure for economic, social and cultural flows, and a site of political contestation. The rapid growth in the number of Internet users into the billions and the importance of networked technologies for most spheres of life has raised questions about how to foster and govern - on a global scale - what has been termed, variously, the 'information society', the 'digital revolution' or the 'information economy'. And these concerns raise questions about power, control and governance. The discovery or creation of new valuable objects - whether we talk about oil and other natural resources, technological infrastructures like the telegraph, or even the New World or outer space - always raises questions about governance and politics. And the Internet is no exception to this rule. When new objects become sites of contestation, questions about governance and power move to the fore: who does this space belong to, what rules and forms of governance should apply, and who should set and enforce them? In the past, the replies to such questions have often revolved around national sovereignty, representative democracy and intergovernmental agreements. But there are also more hybrid forms of governance at work in global politics. Alongside national and intergovernmental forms of steering, we see the 
emergence of new forms of global governance (Held and McGrew 2002), new multilateralisms (Bull 2010), private authority (Hall and Biersteker 2005), and networks involving both public and private actors (Slaughter 2004). At the margins of conventional politics, we see the emergence of novel organizational arrangements that not only make new objects political, but also open up formal politics to contestation and unsettle the boundaries between the economic, the political and the technical. To make sense of the importance of such forms of steering, we need to think of politics as not only a matter of allocating values through formalized and authoritative political systems (Easton 1971), but also of positioning objects as political, and creating sites where such forms of contestation can take place (Barry 2001).

\section{PURPOSE AND FOCUS}

This book examines how and with what implications the global politics of the Internet has been organized around multi-stakeholder processes, under the auspices of the United Nations. Multi-stakeholder arrangements, or what we may term 'hybrid forums' (Callon, Lascoumes et al. 2009), comprising governments, business, technical associations and civil society groups have emerged as a novel way of addressing emergent socio-political problems and opportunities. This book claims that the current shape of the global politics of the Internet is the result of intense and prolonged interactions in such organizational arrangements operating in and around the United Nations. While not necessarily central to formal politics and decision-making as they are played out in, say, national parliaments and the Security Council of the United Nations, hybrid forums do important work in the making and unsettling of politics and governance. While there is a growing interest in the shifting relationship between government and governance (Rosenau and Czempiel 1992), the emergence and workings of such hybrid forms of organization and steering remain under-studied. In particular, we have only just started to make sense of the myriad of transnational networks comprising governments, business and civil society groups currently shaping emergent socio-political problems and opportunities. While such networks play an increasingly important role in global politics and governance, we know very little about the inner workings, organizational strategies and effects of such hybrid arrangements. This book joins other attempts to fill this gap by providing a rare, in-depth, empirical study of the operations and outcomes of UN-driven experiments with hybrid forms of organization and governance in the area of information and communication technologies (ICTs). Puzzled by the proliferation 
of such organizational arrangements in global governance, and based on a rich, multi-sited and longitudinal study, this book offers a detailed account of the inner workings of the hybrid forums that have played a pivotal role in the reconfiguration of the global politics of the digital revolution under the auspices of the UN. It shows how hybrid forums become important to the making of politics and governance because they facilitate what I term collaboration without consensus and entangled authority, and unsettle the boundaries of conventional politics and 'open up new sites and objects of contestation' (Barry 2001: 194).

Reading this book, you will encounter a number of different organizational forms taken by the attempts to address the politics of the digital revolution. But two of these - the UN ICT Task Force and the Working Group on Internet Governance - take centre stage because they laid the foundation for the strong link between hybrid forums and the global politics of the Internet. While also investigating what came before and after these two organizational arrangements, the analyses focus on the practical, yet significant ways in which these early experiments laid the foundations for the entanglement of a techno-political object and an organizational form. Empirically and conceptually, the chapters of the book explore the linkages between an emergent, transnational issue area in global politics, networked forms of organization, and dialogical approaches to governance. The analyses shed light on the practices, actors, controversies, objects of governance, organizational forms, mechanisms and strategies, as well as the technological and regulatory imaginaries, which make up this transnational, socio-political space. ${ }^{1}$ The book shows how multi-stakeholder processes facilitate interactions between very different social worlds, but also challenge established UN practices. Finally, the book offers an overview of the emergent shape of the global politics of the Internet and discusses the effects of multi-stakeholder processes in relation to questions about power and authority.

The book speaks to at least three audiences. Those who are interested in the global politics of information and communication will find a wealth of detail about how this issue area has been shaped by a wide range of actors in recent years. Furthermore, the book offers novel insights into the complex relationships between governance, organization, politics and power, and contributes to emergent, 'alternative' approaches to the study of global governance, politics and regulation. For those who are looking for an analytical vocabulary and a research strategy based on insights from ethnography, actor-network theory, governmentality studies and other situated, process-oriented approaches, this book shows where such analyses may start, what they may yield and what their limits may be. For those involved in organizing initiatives that facilitate interactions 
between different actors, groups, and sectors, this book offers an overview of various techniques and strategies that can be put to work in multistakeholder processes and hybrid forums. Similarly, there are those who may have participated in multi-stakeholder processes in national or international settings, in which case this book offers an invitation to reflect on, and maybe shape, such attempts at organizing hybrid forums and engaging stakeholders in new ways.

\section{THE GLOBAL POLITICS OF THE DIGITAL REVOLUTION}

The global politics of the Internet resembles other attempts at making new objects governable, but it also differs on a number of counts. Unlike many other spaces, the Internet is transnational in scope, boundless in scale, and subject to distributed forms of control (Mueller 2010). The governance of the Internet involves a wide range of activities, from the technical management of domain names and root servers, through intellectual property rights and the regulation of content to questions about access and development. These forms of steering and coordination involve a multitude of public and private actors at both national and international levels. Furthermore, the Internet is subject to such different types of governance as laws, standards, memoranda of understanding, technical coordination and multi-stakeholder dialogues. Furthermore, many forms of regulation are built into the architecture of the Internet and its applications, such as its codes and protocols (Lessig 1999; Galloway 2004). So rather than the ungovernable space imagined by some Internet pioneers (Barlow 1996) and politicians (Clinton 2000), the Internet is not outside the reach of regulation and control, and has in fact always been subject to various forms of governance.

The relationships between information and communication technologies, governance and development have a long history as intergovernmental concerns and are institutionally anchored in international, multilateral bodies. The acceptance of this linking of technological and societal 'imaginaires' (Flichy 2007), and the focus on making such opportunities available to the poorest parts of the world is not new. In particular at the level of national politics, such hopes for technology-driven social transformation have figured prominently for many years. In international politics, this issue has also been discussed frequently and in a number of institutional settings, such as the G8 and the UN, but has gained new momentum at the global level in recent years, particularly under the heading 'the global digital divide'. As Chadwick points out: 
In recent years, a plethora of global information society initiatives has emerged. Some have developed as part of much broader approaches to financial, trade, social, and development issues. Others are highly specific programs in the area of information and communication technologies. But what unites the vast majority of these is the view that the information society is a positive force that should be encouraged in a global context. (Chadwick, 2006: 211)

In the context of the UN, these different aspects of the global politics of the digital revolution have intersected with long-standing concerns about development and human rights, as well as media-specific issues such as access and infrastructure. Importantly, recent initiatives like the United Nations-sponsored World Summit on the Information Society in 2003-05 and a growing number of more focused initiatives have invigorated discussions about the potentials and problems of the digital revolution in relation to social transformations.

An important starting point for this book is that Internet regulation has become tied to attempts at governing the transformations associated with the use and spread of this technological innovation. Thus, rather than thinking of Internet regulation in narrow terms, this book seeks to contribute to the understanding of the contours of what may be termed the global politics of the digital revolution. But what is the digital revolution and how can this be understood as an object of global governance? At the outset, it suffices to point out that the global politics of the digital revolution implies a linking of digital, networked media technologies (such as computers, mobile phones and the Internet) and their (potential and manifest) consequences for social, economic, political and cultural development. To think of such links between the Internet and social transformations as an object of governance implies a broad understanding of governance as a matter of solving socio-political problems and creating socio-political opportunities (Kooiman 2003).

This book shows how two aspects of the problems and opportunities of the digital revolution have taken centre stage in recent UN processes to address the global politics of the digital revolution, namely the global governance of the Internet and the potential of ICTs for development efforts. The socio-political opportunities and problems related to the digital revolution have emerged as a heated political issue at the global level. While the emergence of this political controversy is marked by well-known conflicts between developed and developing countries, hopes for international agreements and attempts at unilateral control, it is also addressed and shaped in novel ways. Most importantly, the global politics of the digital revolution are organized around interactions between a wide range of international organizations, governments, civil society groups and business and technical associations. The main contribution of this book is to 
provide a rich and unique exploration of the multi-stakeholder arrangements that have facilitated the construction of the digital revolution as an object of global governance.

When these UN processes started in the early 2000s, there was very little agreement on key questions about the global politics of the digital revolution. As pointed out by Drake,

The global debate on Internet governance is in a liminal state. There is a total lack of consensus about how to define Internet governance, and about which issues and institutions are and should be involved in what manner. Similarly, there is a lack of agreement as to whether there are significant problems with existing governance mechanisms, and whether there are any pressing but unresolved issues that need to be tackled through international cooperation. Adding to the complexity of the situation, the technological and market environment is changing rapidly, making this a classic example of 'shooting at a moving target,' and some stakeholders' interests and policy preferences are in flux. Indeed, there is fairly widespread uncertainty about how best to proceed, and perhaps even some frustration and burn out among participants whose full engagement will be needed if the policy debate is to achieve anything significant. (Drake 2004: 122)

This book shows how multi-stakeholder arrangements have emerged as an important way of dealing with this lack of shared definitions, institutional anchorage, responsibilities, mechanisms and clear policy preferences in the global debate on the politics of the digital revolution. While priorities continue to differ and conflicts persist, the acceptance of multi-stakeholder participation as the most appropriate mechanism for the facilitation of dialogue and cooperation in this area constitutes a path-breaking development, particularly in an intergovernmental setting such as the UN where national governments are in control.

\section{AN ETHNOGRAPHY OF ORDERING}

In order to examine and conceptualize emergent forms of governance, politics and organization, this book offers an analytical framework that directs attention to everyday practices, associations and processes of ordering. Unlike more conventional approaches, it does not dissect the messy richness of the empirical material - the result of the encounter with a massive international organization such as the UN, years of observations in summits, meetings and working groups, conversations with participants, and piles of documents - into neat variables, theory-driven deductions or structural, social, cultural or other 'outside' explanations. Instead, the book explores the organization of this space of governance at the level of practices and associations. 
This research approach may simply be described as ethnographic, but the book is an ethnographic study of a social and political phenomenon, which is not easily delimited. Anthropological research has tended to study discrete communities and bounded 'fields' (Shore and Wright 1997: 14). In contrast, this book sets out to investigate a social and political space that is not bounded by geography, social unity or the walls of an organization. Unlike more traditional, ethnographic 'fields', this space cuts across many institutions, involves such different people as policy and technical specialists, human rights activists, government officials, business leaders and directors of international organizations, moves from one geographical location to the next, and takes the shape of high politics as well as grassroots activism. In order to be approachable as objects of ethnographic inquiry, such political and social spaces require a 'method for analysing connections between levels and forms of social process and action, and exploring how those processes work in different sites - local, national and global' (Shore and Wright 1997: 14).

To guide the analyses of such dispersed and fluid spaces, I propose that we need a practice-oriented, relational and agnostic research strategy for the study of ordering. This approach brings together insights from Foucauldian work on power and governmentality (Burchell 1991; Larner and Walters 2004), actor-network theory (ANT) (Callon 1986; Law 1994; Latour 2005a) and science and technology studies (STS) (Clarke and Star 2008; Hackett, Amsterdamska et al. 2008) as well as global ethnography (Cunningham 1999; Burawoy 2000) and process-oriented approaches to organization (Czarniawska 2008; Hernes 2008). These theoretical underpinnings will be fleshed out in Chapter 1. At this stage, it suffices to point out that an analysis of ordering implies that we study the everyday ways in which 'particular agents, practices, objects and subjects are assembled at different locales' (Larner and Walters 2004: 11). By foregrounding processes of entanglement and ordering (Law 1994; Latour 2005a), the analyses break with the view of governance and organization as reified entities (Larner and Walters 2004: 11) and refrain from invoking shorthand explanations, such as society, culture or interests. Instead, the focus on ordering invites us to conceive of objects, actors and interests as articulated and constituted in a situated and relational manner, and not given in advance (Hoff 2003). For instance, as pointed out by Latour (1987: 108-9), "'interests" are what lie in between actors and their goals', and are articulated as actors select what, in their own eyes, helps them reach these goals. The concern with ordering also builds on Foucauldian insights about power, agency and the governance of socio-political spaces. For instance, Foucault's famous work on sexuality and madness set out to expose the normalization of distinctions between the heterosexual 
and the homosexual, the sane and the insane. By analysing the forms of knowledge, discourses, subjects, institutions and practices, Foucault sought to denaturalize these forms of social regulation and expose their power effects. Along similar lines, this study sets out to map and denaturalize the practices through which the digital revolution was constructed as an object of global governance and multi-stakeholder arrangements connecting different social worlds were posited as the primary mode of organization in this arena.

Such approaches share the assumptions that processes of ordering involve associations and constellations of human and non-human elements; that they must be investigated at the level of practices; and that they must be understood as fragile and contingent articulations and orderings. To make sense of the implications of this research strategy for the empirical, analytical and conceptual steps taken in this book, it is useful to foreground the three analytical principles around which it revolves.

\section{Three Analytical Principles}

Most studies of global governance have little interest in situated practices, give ontological priority to entities rather than associations, and tend to start from pre-defined categories and objects. In contrast, this book pursues the study of global politics, governance, and organization at the level of practices, focuses on relations, and proceeds in an agnostic manner. Let us look briefly at the implications of the three analytical principles.

The first analytical principle underpinning this study is that the smallest unit of analysis is that of practices, understood as 'organized sayings and doings' (Nicolini 2009: 1401; Orr 1998). Such mundane ways of doing things together (Clarke and Star 2008) may take the shape of discursive and other practices, such as conducting a meeting, interacting in an online forum or building and running an organization. As Nicolini (2009: 1400) points out, 'When it comes to practice, distinguishing material and discursive aspects only has an analytical, not a substantive value, and one should not be given ontological priority over the other.' Starting the analyses from practices allows us to demonstrate how myriads of mundane practices and associations produce what we normally think of as 'society', 'governance', 'an organization' or an 'issue area'. The orientation to practices is central to an ordering perspective because it means '[w]e start from "irreducible, incommensurable, unconnected localities, which then, at great price, sometimes end into provisionally commensurable connections"' (Kendall 2004: 73, quoting Latour 1997).

Practice-oriented studies are rare in the literature on governance and global politics, and sociological and ethnographic approaches still operate 
at the margins of disciplines like international relations (IR). Still, we have some proponents of what may be termed a 'practice turn', including Laffey and Weldes's (2005) work on the practices of 'policing', Bigo and Walker's (2007) discussion of the 'international' as practices, Sending and Neumann's (2006; Neumann and Sending 2007) writings on governmentality and global governance, and Doty's (1997) discussion of how the attention to practices may move us beyond the agent-structure problematique in IR theory. In political science more broadly, the works of Bevir and Rhodes (2003), Flyvbjerg (2001), Schram and Caterino (2006) and Hajer and Wagenaar (2003) have established practices as an important entry point for studies of politics, power and governance. Finally, the concern with practices has been fuelled by scholars seeking to bring ethnographic approaches into IR theory, such as when Mandaville (2002) stresses the value of anthropological readings of, for instance, the nationstate. Such studies tie in with the call for practices to move centre stage, and shed light on the sites and situations where governance and politics are articulated and carried out.

Second, this book is underpinned by an analytical principle that may be termed relational. A relational approach urges us to refrain from thinking in terms of discrete entities, and instead unpack associations. While studies of global governance explore the regulatory and authoritative relationships that state and non-state actors develop and institutionalize, most conventional approaches nevertheless consider relationships to be composed of pre-constituted entities. While various forms of (new and old) institutional theory (Gössling, Oerlemans et al. 2007), social network theory (Jones, Hesterley et al. 1997; Wasserman and Faust 1994) and transaction cost economics (Dekker 2004) may study relations, they do so from a substantialist focus on entities, rather than a relational perspective considering the social world to consist of dynamic and unfolding processes (Emirbayer 1997). That is, they reflect the assumption that 'entities precede interaction, or that entities are already entities before they enter into social relations with other entities' (Jackson and Nexon 1999: 293). In contrast, a relational approach implies the view that 'the very terms or units involved in a transaction derive their meaning, significance, and identity from the (changing) functional roles they play within that transaction. The latter, seen as a dynamic, unfolding process, becomes the primary unit of analysis rather than the constituent elements themselves' (Emirbayer 1997: 287). As an analytical principle, a relational approach implies that we give primary attention to the 'constitutive relations' (Barnett and Duvall 2005: 9) where the various elements of governance - objects, procedures, subjects, power and knowledge - are established, negotiated and challenged.

The question of whether we consider the social world to consist of enti- 
ties or processes, in 'static "things" or in dynamic, unfolding relations' (Emirbayer 1997: 281) is a well-established debate, just like the argument that we need to study power, identities, interests, technology and other phenomena in a relational manner has been made consistently and in multiple disciplines. For instance, such perspectives stress that identities are constituted in interactions and can only be understood in the context of the relations through which they are realized, negotiated and contested (Hylland Eriksen 1999). Or that power is not a resource or a thing simply possessed by some and wanted by others, but the product of relationships in particular sites (Foucault 1977b: 122). Still, such 'postfoundational' (Bevir 2004: 608) and 'radical decentered' (Doty 1997: 376) insights remain under-explored in studies of governance, networks and power.

The third analytical principle is that the analyses have an agnostic starting point. As a research strategy, an agnostic approach implies that we do not 'decide in advance what the phenomenon consists of on the basis of prior formal analytic studies' (Garfinkel and Rawls 2002: 171). In line with ethnography, ethnomethodology and actor-network theory, an agnostic approach sets out to 'follow the actors' (Latour 2005a) without being 'conceptually committed to [. . .] prior objects' (Larner and Walters 2004: 5), such as theoretical, managerial, structural, social or other 'outside' explanations (Latour 2005a; Clarke 2005). So to be agnostic 'entails a move of "bracketing" the world of underlying forces and causes, and instead examining the different ways in which the real has been inscribed in thought' (Larner and Walters 2004: 16). By not settling on the shape of, for instance, power and networks in advance it is possible to foreground the making and ordering of actors, issue areas and organizational arrangements, rather than taking them for granted.

In contrast, conventional approaches tend to start from analytical units such as 'structures', 'institutions' or 'ideas', or to define some factors as independent variables and others as dependent ones. But as Bevir (2004: 609) points out: 'Aggregate concepts, such as a class or an institution, cannot be adequate markers for people's beliefs, interests or actions. Such aggregate concepts stand only as abstractions based on the multiple and complex beliefs and actions of the individuals we locate under them.' To be agnostic about the shape and locations of power allows us to explore the operations of power in all (potentially significant) aspects and processes of dialogue about the global politics of the digital revolution. It provides an alternative to the view of institutional arrangements as monolithic structures, and invites us to investigate them more openly - as contingent assemblages under constant (re)construction. Such agnostic approaches explore how interests, authorities and identities are articulated and stabilized through linkages in concrete situations. 
These principles have consequences for how we approach the empirical material, engage theoretical insights and conceptualize governance, organization and power. This study sets out to map the assemblages and emergent orderings that make up the organizational forms, governance discussions, and forms of authority involved in the global politics of the Internet. Along the lines of governmentality studies and actor-network theory, we may think of this techno-political space as a complex web of humans, non-humans, discourses, processes, positions and ideas. To approach governance and organization as practical associations among different elements in temporally and spatially demarcated situations has a number of resemblances to what Clarke (2005) terms 'situational analysis' - a poststructuralist rethinking of grounded theory. Following the Straussian line in grounded theory, she shows how social formations can be studied in a situated, relational manner. One important insight is to refrain from thinking about 'context' as something outside our objects of investigation: "The conditions of the situation are already in the situation. There is no such thing as "context". The conditional elements of the situation need to be specified in the analysis of the situation itself as they are constitutive of it, not merely surrounding it or framing it or contributing to it. They are it' (Clarke 2005: 71-2). By studying entanglements of elements from within situations, Clarke shows how we can study events and their conditions as one, and thus capture the social formations, positions, discourses and other complexities of situations. Although not framed as a form of grounded theory, the present study has many affinities with Clarke's situational analysis and carries out similar analytical operations.

\section{THE BOOK AHEAD}

The first chapter advances the concept of ordering and situates the book in relation to the existing literature on governance, organization and power in global politics. Proposing that ordering is best understood in terms of assemblages and translations, the chapter fleshes out these two analytical concepts and shows how they are engaged in this study of hybrid forums and the global politics of the Internet. Paving the way for analyses of the ordering of this emergent space of governance, the chapter goes on to outline four significant effects of ordering, namely the making, shaping and stabilization of subjects, objects of governance, political rationalities and organizational techniques. Finally, the chapter proposes that ordering is ultimately about power, and suggests how theories about power, authority and control may guide the conceptualization of the effects of ordering on subjects, objects, techniques and rationalities. Chapter 2 launches the 
empirical analysis of the first attempts at ordering the global politics of the digital revolution. It shows how this was driven by a strong belief in the ability of technology to spur social transformation, and captures the ways in which this emergent space of governance has been problematized and shaped through experiments with multi-stakeholder processes in and around the UN system. Chapter 3 shifts the attention to the enrolment of stakeholders as a key part of ordering in hybrid forums. Focusing on processes of inclusion, exclusion and categorization, this chapter provides insights into the power effects of engaging social worlds as stakeholders. Chapter 4 focuses on the organization of hybrid forums in dialogues about the global politics of the Internet. It provides a rich account of the positioning, structuring and steering of multi-stakeholder processes, with particular attention to the multiple organizational techniques used to make members work together and connect with broader groups of stakeholders. Chapter 5 focuses on the ability of hybrid forums to position themselves as platforms for dialogue about the global politics of the digital revolution, and shows how they have ordered this object of governance in new ways. Chapter 6 addresses the ways in which multi-stakeholder processes to address the politics of the Internet continue to take new shapes and directions. Finally, the conclusion conceptualizes the power of hybrid forums and the effects of governing through dialogue for political subjects, objects of governance, organizational techniques and political rationalities. 
Mikkel Flyverbom - 9780857936462 Downloaded from PubFactory at 04/26/2023 10:19:12AM via free access 